\title{
Cambio en los patrones de crecimiento en una ciudad intermedia: el caso de Chillán en Chile Central
}

\begin{abstract}
The patterns of urban growth of Chillán city (Central Chile), the land use associated to each of them and the factors which guide the urban expansion process are identified. Changes in the built urban surface during a period of 57 years (from 1943 until 2000) were quantified. Between 1997 and 2000 were analyzed the changes of land use, population density and home socioeconomic conditions, utilizing aerial photographs, SIG (Arcview 3.2 and Arc-Info N.T) and Spatially Explicit Landscape Index. A regression analysis allowed to study the relation between urban growth and population growth.
\end{abstract}

Key words: urban growth, Spatially Index, SIG, Chillán City.

\section{Resumen}

Se identifican los patrones del crecimiento urbano de la ciudad de Chillán (Chile Central), el uso del suelo asociado a cada uno de ellos y los factores que guían el proceso de expansión urbana. Se cuantificaron los cambios en la superficie urbana construida en un período de 57 años (de 1943 hasta el 2000). Entre 1997 y el año 2000 , se analizó el cambio en relación con el uso del suelo, la densidad de población y la condición socio-económica de los hogares. Se utilizaron fotografías aéreas, SIG (Arcview 3.2 y Arc-Info N.T) e índice de análisis espacial (Spatially Explicit Landscape Index). Un análisis de regresión permitió estudiar la relación entre crecimiento urbano y crecimiento de población.

Palabras claves: crecimiento urbano, índices espaciales, SIG, ciudad de Chillán. 


\section{Introducción}

a ciudad es el hábitat humano por excelencia; la proporción de población urbana en comparación con la población rural y el crecimiento acelerado de las ciudades, tanto en habitantes como en extensión, son fenómenos globales que así lo demuestran. La región latinoamericana, y Chile en particular, han sido afectados notablemente por el crecimiento urbano. El Censo de Población y Vivienda realizado en 2002 revela que el $87 \%$ de la población chilena vive en áreas urbanas, y que la aceleración del crecimiento urbano no es sólo un fenómeno propio de la ciudad capital o de las grandes ciudades, sino que se extiende por todo el territorio, y es observable en las ciudades intermedias del país y centros administrativos menores.

Explicar cómo ha ocurrido este proceso y cuál ha sido el comportamiento de las ciudades frente al crecimiento, ha motivado una preocupación cada vez mayor de cientistas sociales, arquitectos y geógrafos. En general, de los trabajos recientemente publicados se desprende que los impactos de la economía de mercado, a nivel local y regional, el comercio y la movilidad de la población son las fuerzas que guían los procesos de expansión urbana, y que la respuesta de las ciudades ha sido, muchas veces, un crecimiento explosivo y una tendencia a la fragmentación y segmentación social del espacio urbano y uniformidad de paisajes (Vink, 1982; Romero \& Toledo, 2000; Mertins, 2000).

Si bien existen factores globales que explican la evolución reciente de las ciudades intermedias latinoamericanas en un contexto de

Unidad de Planificación Territorial, Centro EULA-Chile, Universidad de Concepción. Casilla 160C, Concepción, Chile. E-mail: gazocar@udec.cl y rsanhue@udec.cl

* Instituto de Geografía, Pontificia Universidad Católica de Chile. Doctorado en Ciencias Ambientales Centro EULA-Chile, Universidad de Concepción. E-mail: cghenriq@puc.cl creciente globalización económica, también es posible encontrar factores locales y regionales que les otorgan rasgos particulares a los procesos de desarrollo urbano. Dentro de los factores locales encontramos la situación socioeconómica de la población, que incluye procesos demográficos, status social, nivel educacional e ingresos, stocks de viviendas y políticas de uso del suelo, con especial atención en la regulación de zonas urbanas (Young, 1995), movimientos intraurbanos de grupos sociales y la continuidad de las migraciones desde el campo hacia la ciudad (López et al., 2001).

Los factores regionales están más vinculados con la posición geográfica del centro urbano, en relación con los mercados de trabajo, infraestructura de transporte y servicios (McDonal \& McMillen, 1998).

En muchas ciudades de América Latina, el surgimiento de nuevas zonas residenciales, comercios, equipamientos y servicios destinados a grupos sociales cada vez más segregados espacialmente ha sido un importante factor de crecimiento urbano que, en muchos casos, parece ser cada vez más dependiente de la presencia de una élite de altos ingresos, que no supera al $10 \%$ de la población y que varía substancialmente entre una ciudad y otra (Romero \& Toledo, 1998a). En este sentido, algunos autores señalan que los nuevos procesos de urbanización no sólo han provocado cambios físicos en la morfología urbana (Barber, 2000; De Mattos, 2001), sino que además han acrecentado las brechas o desigualdades socioeconómicas dentro de las ciudades, con un aumento de la polarización y de la fragmentación del espacio urbano (Hanhörster, 2001; Sabatini, 1998; Romero \& Toledo, 1998b; Azócar et al., 2003).

En Chile, investigaciones realizadas en Valdivia (Borsdorf, 2000), Puerto Montt (Rovira, 2000), Temuco (Romero \& Toledo, 2000) y Los Angeles (Azócar et al., 2003), coinciden en la importancia de las ciudades intermedias como centros administrativos regionales y provincia- 
les, como centros prestadores de servicios para sus hinterlands y centros de transformación industrial de recursos naturales. Son ciudades que viven un despegue económico originado por la localización, en su ámbito territorial, de inversiones orientadas al mercado internacional, principalmente forestales, pesca, cultivos acuícolas y turismo especializado, constituyéndose en verdaderos nodos de una estructura reticular que, progresivamente, las ha transformado en centros regionales de distribución y consumo de complejas redes bancarias, financieras, comerciales, educacionales y sanitarias (Romero \& Toledo, 2000).

Para Boisier (2001), las ciudades han asumido nuevas funciones en el actual modelo económico, y operan como lugares centrales desde donde se organiza la economía de una región o territorio, transformándose en puntos claves para la localización de servicios especializados para la producción, así como en nodos generadores de innovaciones.

En el caso de Chile, no existe suficiente evidencia empírica que permita conocer científicamente la evolución de los patrones de urbanización y sus factores causales, como tampoco sus impactos sobre la morfología y el reordenamiento social del espacio urbano. Sin embargo, investigaciones recientes nos indican que aún estamos en presencia de los denominados ejes radiales y sectoriales del desarrollo y expansión urbana, modelos tradicionales desarrollados para explicar la organización del espacio urbano en las ciudades latinoamericanas (De Mattos, 2002). En estos mismos trabajos se mencionan nuevos procesos y estructuras espaciales que estarían orientando las transformaciones urbanas, como las denominadas estructuras insulares de la ciudad y la ocurrencia, en zonas urbanas que alguna vez fueron más homogéneas, de procesos contradictorios como valorización, aislamiento y privatización (Hervieu \& Viard, 1996; Thomson \& Mitchell, 1996).

En este contexto, el estudio de los factores que orientan el crecimiento urbano en una ciudad intermedia de Chile Central es uno de los objetivos principales de esta investigación, con especial énfasis en el análisis de los factores que inciden en los cambios en los patrones de uso del suelo y su relación con la evolución de la población y su condición socioeconómica. La ciudad de Chillán es un núcleo administrativo de nivel provincial, que ejerce una fuerte atracción sobre el sistema de asentamientos urbanos de la provincia de Ñuble y sobre el entorno agrario que la rodea, prestando servicios de apoyo a actividades productivas y constituyendo un foco de atracción para la localización de equipamientos, infraestructuras y el asentamiento de población.

\section{Objetivo y métodos}

El objetivo de esta investigación es analizar el crecimiento urbano de la ciudad intermedia de Chillán en el periodo 1943-2000, identificando los patrones espaciales y temporales asociados a los cambios en el uso del suelo. El método se basa en tres lecturas: (a) observaciones en terreno; (b) análisis espacial del cambio de la superficie urbana construida y del uso de la tierra y (c) análisis de los cambios sociodemográficos y residenciales. Se identifican los patrones temporales y espaciales de este crecimiento y sus factores causales según diferentes contextos del paisaje, uso del suelo asociado y factores que han activado la expansión.

\subsection{Cuantificación del cambio urbano}

Se cuantificaron los cambios en la superficie urbana construida en un período de 57 años, para lo cual se utilizaron fotografías aéreas, SIG (Arcview 3.2 y Arc-Info N.T) y se aplicó un índice de análisis espacial "Spatially Explicit Landscape Index" (SELI) que permite conocer las distancias del cambio urbano en relación con la condición de ciudad o no ciudad en cada uno de los períodos considerados (Wiegand, 2001). Entre los años 1997 y 2000 se hace un análisis 
más detallado del cambio urbano para diferente usos del suelo, densidad de población y condición socioeconómica de los hogares.

La información obtenida permitió construir las coberturas de perímetro urbano, usos del suelo, zonas con hogares en diferente condición socioeconómica y densidad de población. Con estas variables se analizaron las tendencias del proceso de expansión urbana y se investigaron los factores que explican el crecimiento de Chillán, con especial énfasis en la identificación de patrones de crecimiento y su relación con la distribución de la población en la interfase urbano-rural, los procesos de recomposición social y el contexto específico de paisaje en que se produce el cambio.

\subsection{Construcción de las zonas socio- económicas}

Para construir las zonas socioeconómicas de Chillán se utilizaron los datos del Censo de Población y Vivienda del año 1992 (INE, 1992), procesando la información en el programa REDATAM-Plus y enlazando la base de datos censal con el SIG (Arcview 3.2). Se trabajó la información censal a nivel de manzana y se aplicó el indicador denominado Necesidades Básicas Insatisfechas (NBI), según la metodología de CEPAL. Las variables utilizadas para medir las carencias físicas en el hogar fueron estructura de la vivienda; abastecimiento de agua potable; disponibilidad de servicio sanitario y hacinamiento, mientras que las variables utilizadas para medir la vulnerabilidad fueron la tasa de dependencia económica y nivel educacional del jefe de hogar. El cruce de ambos índices carencias físicas y vulnerabilidad- permitió clasificar los hogares de Chillán en zonas críticas, pobres, vulnerables, mixtas y no pobres (Henríquez \& Azócar, 2001).

\section{Resultados}

\subsection{El área de estudio}

La ciudad de Chillán se ubica en la depresión intermedia de Chile Central, próxima a Concepción, capital de la región del Biobío, y a cuatrocientos kilómetros de la ciudad de Santiago (Figura 1). La población comunal asciende a 156.537 habitantes, de los cuales el $88 \%$ se concentra en la ciudad de Chillán y un $12 \%$ en las zonas rurales de la comuna (INE \& MINVU, 2001).

Chillán es una ciudad intermedia que en las últimas décadas ha experimentado un fuerte crecimiento urbano y demográfico, doblando su población entre los años 1952 y 1982, como también su trama urbana (Olave, 1994 y 1996). En el último período intercensal, entre 1982 y 1992, presentó una tasa de crecimiento de un $2 \%$, superior a la media regional, de 1,3\%, y nacional, de 1,6\% (INE, 1992; Olave, 1996; González et al., 1994; Olave et al., 1996).

\subsection{Uso del suelo urbano}

En la estructuración física de Chillán se distinguen varias macrozonas: el núcleo urbano central, las áreas periféricas y el área de extensión en torno al Bypass. La parte central es un sector de trama regular originada en la cuadrícula histórica de la ciudad y organizada a partir de los equipamientos, servicios y funciones que allí se localizan (Azócar \& Muñoz, 1998). La periferia adopta forma de anillos concéntricos, equidistantes del área central, con sectores urbanos de diferentes tamaños e irregular distribución espacial, cuyo origen ha sido el crecimiento del casco urbano sobre terrenos agrícolas (Azócar \& Muñoz, 1998). La macrozona de extensión se localiza en el costado poniente de la ciudad y se desarrolla linealmente, siguiendo el eje del Bypass de la Ruta 5 Sur o Carretera Panamericana. 
Figura 1. Área de estudio.

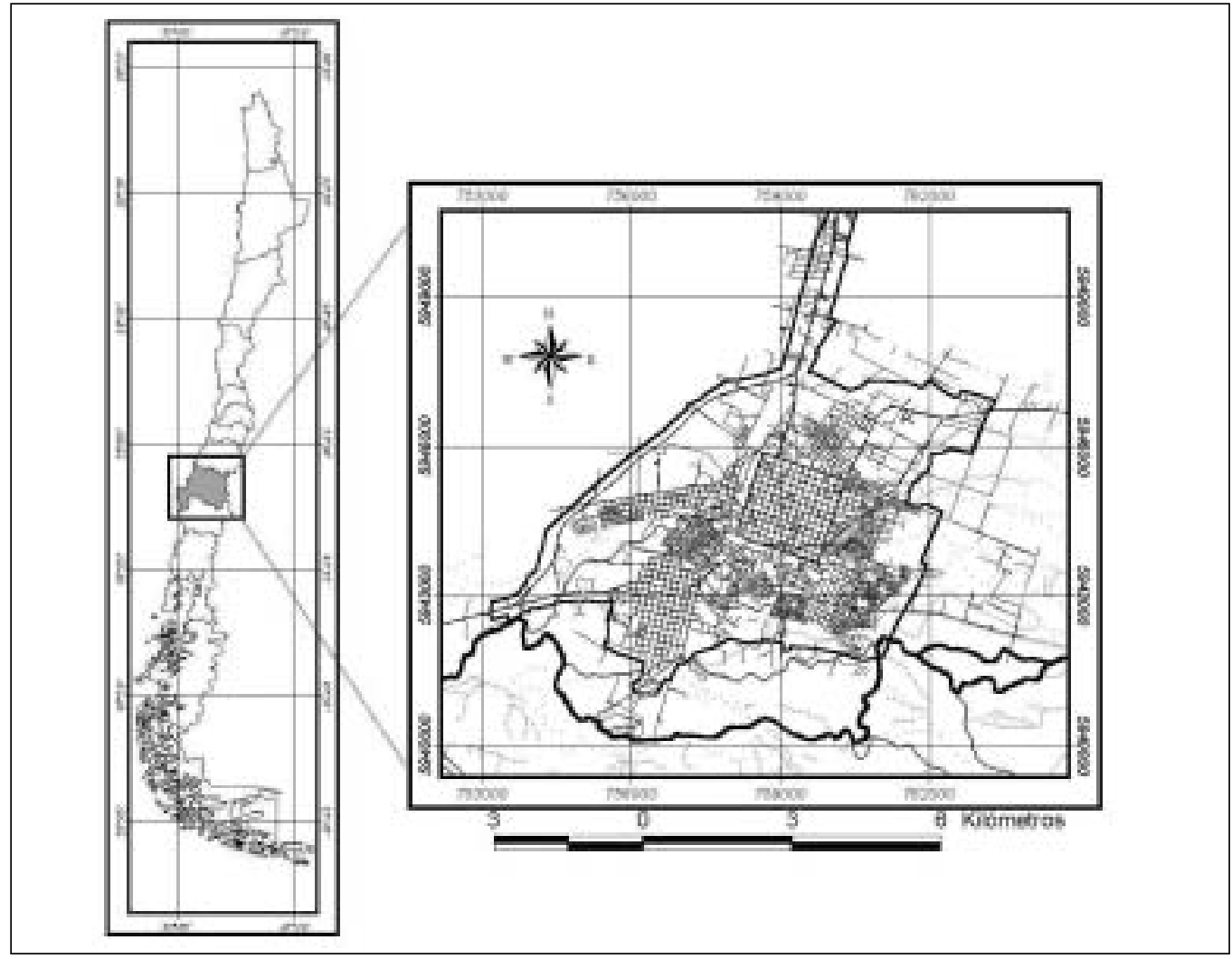

Todas estas macrozonas están comprendidas dentro del límite urbano de Chillán, perímetro que posee un contorno irregular y que abarca una mayor superficie en las proximidades de las vías que comunican la ciudad con el resto de la comuna. Los usos del suelo urbanos, permitidos en el actual Plan Regulador, son los siguientes: zonas urbanas $(2.203,3$ hectáreas), zonas especiales (1.737,3 hectáreas) y zonas de restricción (245,3 hectáreas).

En las zonas urbanas el uso predominante es vivienda con equipamiento, talleres y almacenamiento inofensivo. En el sector periférico es posible distinguir zonas especiales que, a diferencia de la anterior, permiten la localización de industria molesta y presentan una menor densidad de ocupación del suelo. En esta parte de la ciudad se ha observado en los últimos años un proceso sostenido de expansión urbana, cuyos efectos sociales se pretenden evaluar en este trabajo. Antecedentes preliminares, obtenidos en terreno, nos informan de la alteración de cuerpos de agua y de la pérdida de suelos agrícolas, como también del surgimiento de áreas marginales o slums ubicadas dentro de los límites urbanos y en la interfase urbano-rural.

Las zonas de restricción se delimitan básicamente por la presencia de infraestructura vial y energética. Al parecer, en la delimitación de estas áreas no fueron considerados los riesgos naturales, como tampoco la protección de suelos agrícolas o zonas de alto valor ecológico, como humedales o cauces de esteros. Olave (1996) señala que Chillán se caracteriza fundamentalmente por presentar una construcción continua y por un proceso de expansión urbana gatillado por el crecimiento de la población, así como también por las migraciones rural-urbanas, con fuertes impactos sobre la actividad residencial y los servicios. 


\subsection{El crecimiento urbano y demográfico en Chillán}

Para estimar el crecimiento urbano de Chillán en función del crecimiento de la población se construyó una función de predicción de la superficie urbana construida entre los años 1943 y 2000, en base a los valores observados de superficie urbana construida, obtenido de fotografías aéreas para distintos períodos, y los datos de población de los Censos de Población y Vivienda (Cuadro 1).

Cuadro 1. Valores observados y estimados de superficie urbana construida y población de Chillán (19432000).

\begin{tabular}{|c|c|c|c|c|}
\hline Año & $\begin{array}{l}\text { Superficie urbana } \\
\text { construida }^{\text {(a) }} \text { (há) }\end{array}$ & $\begin{array}{l}\text { Superficie urbana } \\
\text { estimada }^{(b)} \text { (há) }\end{array}$ & $\begin{array}{l}\text { Diferencia } \\
\text { (há) }\end{array}$ & $\begin{array}{l}\text { Población }{ }^{(\mathrm{c})} \\
\text { (miles habitantes) }^{\text {(mila }}\end{array}$ \\
\hline 1943 & 830,99 & 806,95 & 24,04 & 45.073 \\
\hline 1955 & 927,35 & 949,71 & $-22,36$ & 56.969 \\
\hline 1961 & $1.045,59$ & $1.071,03$ & $-25,44$ & 67.079 \\
\hline 1978 & $1.518,89$ & $1.549,55$ & $-30,66$ & 106.956 \\
\hline 1991 & $1.796,58$ & $1.978,94$ & $-182,36$ & 142.738 \\
\hline 1997 & $2.097,01$ & $2.199,9$ & $-102,89$ & 161.154 \\
\hline 2000 & $2.443,31$ & $2.361,67$ & 81,64 & 174.632 \\
\hline
\end{tabular}

(a) Valores calculados en Arcview 3.2 a partir de las coberturas temáticas de espacio urbano construido. Estas cubiertas fueron elaboradas digitalmente, en base a fotografías aéreas georreferenciadas.

(b) Valores estimados de superficie urbana modelo predictivo y $=266,082+0,012^{\prime} x$. Fuente: elaboración propia.

(c) Estimación sobre la base de los Censos de Población y Vivienda del Instituto Nacional de Estadística de los años 1940, 1952, 1960, 1970, 1982 y 1992 (INE).

La estimación de población se realizó con la aplicación del modelo de crecimiento geométrico:

$$
\text { donde: } r=\left\{\left(\frac{N x}{N o}\right)^{1 / t}-1\right\} * 100
$$

$$
\begin{aligned}
\mathbf{r} & =\text { tasa de crecimiento intercensal; } \\
\mathbf{N x} & =\text { población en un año } \mathbf{x} ; \\
\mathbf{N o} & =\text { población en el año anterior a } \mathbf{x} ; \mathbf{y} \\
\mathbf{t} & =\text { tiempo transcurrido en el período }(\mathbf{x}- \\
& \mathbf{0}),
\end{aligned}
$$

según la función de predicción o modelo lineal: $y=266,082+0,012^{\prime} x$ donde:

$$
\begin{aligned}
& \mathbf{y}=\quad \text { crecimiento urbano estimado (há) } \\
& \mathbf{x}=\begin{array}{l}
\text { población estimada (miles de habi- } \\
\text { tantes) }
\end{array}
\end{aligned}
$$

El crecimiento del área urbana de Chillán hasta la década del ' 80 puede ser explicado por el crecimiento de la población. Los valores obtenidos en el modelo, para los valores comprendidos entre los años 1943 y 2000, fueron: $r=0.991, r^{2}=0.982$ y $^{2}$ ajustado $=0,978$. Como se observa en la Figura 2, a partir de un punto de la curva el cambio no es constante y el crecimiento de la población urbana de Chillán no explica el fuerte incremento en la superficie urbana construida, mostrando un crecimiento urbano de tipo exponencial. 
Entre 1991 y el año 2000 la población de la ciudad pasó de 142.738 a 174.632 personas, con una tasa de crecimiento cercana a un $2 \%$ anual, mientras en igual período, la tasa de construcción de viviendas pasó de un $18,5 \%$ a un $35,9 \%$, pasando de 34.283 a 46.580 viviendas construidas (INE, 1992; INE \& MINVU, 2001). Lo anterior estaría indicando que el proceso de crecimiento urbano de Chillán a partir de la década del '80 estaría más vinculado con el surgimiento de nuevos barrios en zonas periféricas de la ciudad, y que el crecimiento poblacional no es un factor explicativo del incremento de la superficie urbana construida. El reemplazo de viviendas de emergencia en sectores urbanos en situación irregular por las denominadas viviendas básicas explicaría, en parte, estos nuevos desarrollos inmobiliarios para sectores de bajos ingresos.

Figura 2. Relación entre crecimiento de la población y área urbana de Chillán (período 1943-2000).

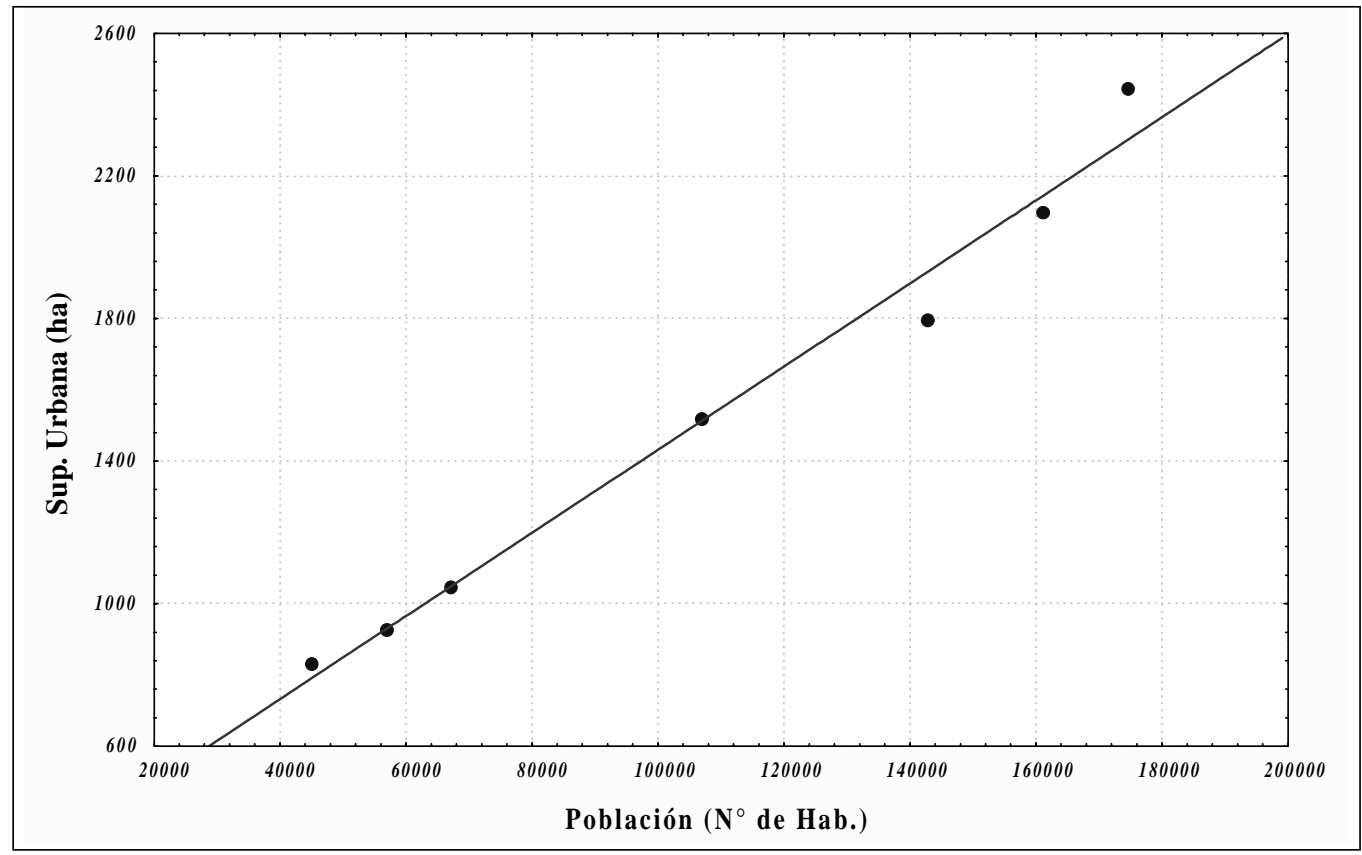

Entre 1943 y el año 2000 la ciudad de Chillán triplicó su tamaño, pasando de 830 hectáreas a 2.443,3 hectáreas. Entre 1943 y 1961 la ciudad creció, en promedio, 12 hectáreas por año, y entre 1961 y 1991 presentó un crecimiento mayor que en décadas anteriores, con tasas mayores a 20 y cerca de 25 hectáreas por año. A partir de 1990 se produce un notorio incremento en la superficie urbana construida, con un consumo promedio, hasta el año 1997, de 43 hectáreas por año.

La tendencia observada hasta la década del ' 80 corresponde a un crecimiento urbano lineal fuertemente relacionado con el incremen- to de la población, el cual da paso a un crecimiento exponencial vinculado a otros factores, con tasas que superan las 100 hectáreas por año en los últimos años (Figura 2). Las proyecciones contenidas en los instrumentos de planificación refuerzan la tendencia a este tipo de crecimiento, con previsiones de incorporar anualmente, entre los años 2000 y 2010, 153,6 hectáreas de nuevos suelos urbanos.

\subsection{Los patrones del crecimiento}

La secuencia del cambio urbano entre los años 1943 y 2000 se obtuvo del análisis de las celdas o pixeles de la matriz perímetro ur- 
bano construido que cambiaron, en ese período, su condición de no ciudad a ciudad (Cuadro 2). Con la aplicación del "Spatially Explicit Landscape Index" (SELI) se conocieron las distancias medias del crecimiento urbano para cada uno de los períodos analizados.

Entre 1943 y 1955 la ciudad de Chillán se extiende, en todo su perímetro urbano construido, a distancias inferiores a doscientos metros. Distancias mayores no son significativas. Entre la década de 1940 y hasta 1960, el crecimiento tendrá un comportamiento constante, con una tasa inferior a 20 hectáreas por año y con distancias al perímetro urbano construido inferiores a 200 metros. Se trata de un crecimiento en forma de anillos (ribbonurbanization) asociado a nuevas zonas residenciales. Entre 1978 y 1991 el número de celdas con cambio urbano se triplica respecto al período 1943-1955, como también la distribución de las frecuencias. La ciudad, en algunas zonas, crece a distancias mayores a doscientos metros y menores a cuatrocientos. El patrón de crecimiento concéntrico o radial (Zhang, 2001) se mantiene y es posible observar una nueva tendencia: un crecimiento de la ciudad a mayores distancias, relacionado con ejes de transporte, tipo tentacular.

Entre 1997 y el año 2000 el análisis del cambio urbano confirma el planteamiento de un doble patrón de crecimiento. En este período Chillán crece, en algunos sectores, hasta distancias de 1,7 kilómetros, y en la mayor parte del perímetro construido, a distancias inferiores a trescientos metros. Estamos frente a una ciudad que crece en tamaño por agregación de nuevos suelos urbanos, anteriormente rurales, en torno al espacio urbano precedentemente construido, y también a lo largo de ejes de transporte que separan cada vez más el núcleo urbano de las nuevas zonas industriales y residenciales.

Este último patrón de crecimiento, a través de ejes, nos enfrenta a una forma urbana tentacular, en la cual los procesos de cambio más reciente han sido tensionados por las infraestructuras de transporte y los efectos que ellas conllevan en la localización industrial, en los nuevos barrios residenciales, en los servicios y equipamientos (Figuras 3, 4 y 5).

También es posible identificar un tercer tipo de patrón de crecimiento urbano, que se ha denominado "salto de rana" (leap-frog). En efecto, entre 1991 y 1997 Chillán continuó, en la parte noreste, con su patrón de crecimiento en torno a ejes de transporte, dejando espacios de ruralidad a medida que se extendía. Sin embargo, ahora se van configurando nuevos barrios residenciales o islas urbanas, separadas del perímetro urbano, orientadas a grupos sociales medios-altos. De esta forma, se van configurando pequeñas áreas urbanas alejadas del perímetro urbano en las inmediaciones rurales y también islas de ruralidad en las cercanías del casco urbano consolidado.

Cuadro 2. Matriz de cambio urbano de Chillán entre los años 1943 y 2000.

\begin{tabular}{|lllllll|}
\hline \multirow{2}{*}{ Elemento } & Períodos & & & \\
\cline { 2 - 6 } & $\mathbf{1 9 4 3 -}$ & $\mathbf{1 9 5 5 -}$ & $\mathbf{1 9 6 1 -}$ & $\mathbf{1 9 7 8 -}$ & $\mathbf{1 9 9 1 -}$ & $\mathbf{1 9 9 7 -}$ \\
& $\mathbf{1 9 5 5}$ & $\mathbf{1 9 6 1}$ & $\mathbf{1 9 7 8}$ & $\mathbf{1 9 9 1}$ & $\mathbf{1 9 9 7}$ & $\mathbf{2 0 0 0}$ \\
\hline Celdas con cambio ${ }^{(*)}$ & 90 & 121 & 483 & 274 & 299 & 352 \\
Superficie cambio (há) & 96,4 & 118,2 & 472 & 277,3 & 300,4 & 348,3 \\
Promedio cambio (há) & 8 & 19,7 & 27,8 & 21,4 & 50 & 116,1 \\
Promedio distancia (m) $\left.{ }^{* *}\right)$ & 160,8 & 190,4 & 230,6 & 200 & 400,1 & 420,5 \\
\hline
\end{tabular}

(") Celdas que en el período pasaron de no ciudad a ciudad.

(*) Valores obtenidos con Índice de distancia SELI.

Nota: El índice de distancia calcula la mínima distancia al perímetro urbano construido o alguna de las cubiertas temáticas (valores $>0$ ) para cada una de las celdas con o sin cambio. 
Figura 3. Crecimiento urbano de Chillán 1943-2001.

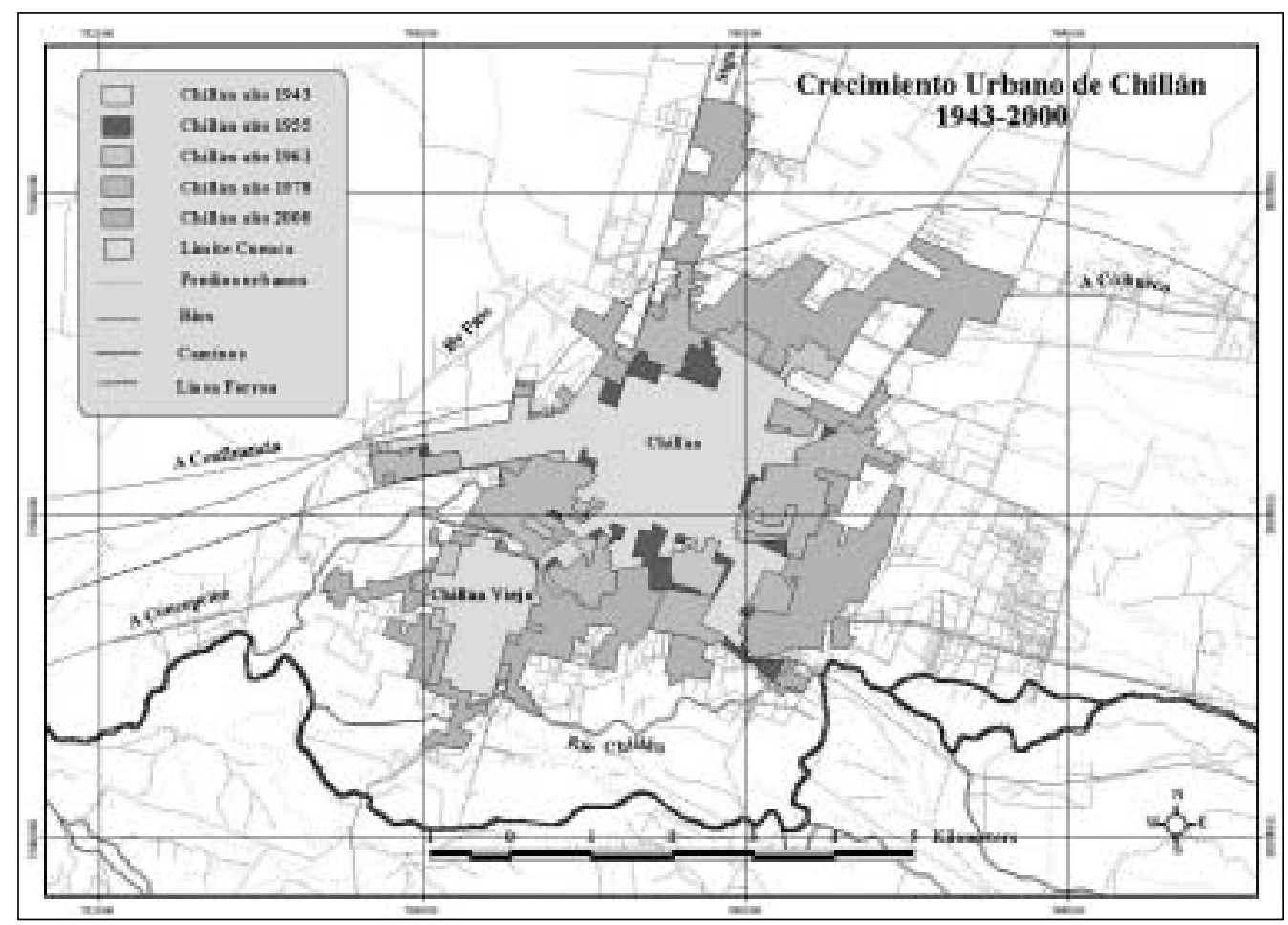

Es interesante destacar la dinámica del cambio en esta parte de la ciudad, por cuanto existe una estrecha relación entre las características del entorno rural inmediato y las condiciones socioambientales que esta zona ofrece, constituyendo un factor local de atracción a nuevas localizaciones residenciales y equipamientos.

Se trata de áreas de crecimiento preferencial, en la cual los agentes privados del desarrollo inmobiliario ofrecen un concepto diferente de ciudad -o más bien de suburbio urbano-, más alejado del centro y de sus clásicos problemas, en un entorno semi-rural o semi-urbano con buena accesibilidad, equipamiento y ambiente natural. Las densidades de población son bajas, entre 0,1 - 50 hab/há, y es el área donde se materializan los principales proyectos de subdivisión o loteos urbanos, cuyas superficies varían entre 0,5 y 1 hectárea.
Las fuerzas del mercado han producido nuevos barrios en esa zona. La comunidad o segmento elegido presenta el siguiente perfil: valores altos de propiedad, altos ingresos de los residentes, alta calidad de equipamiento educacional, grandes tamaños en los lotes y un grupo social homogéneo. Es decir, en esta área estamos en presencia de las denominadas "comunidades atractivas" (Hervieu \& Viard, 1996; Thomson \& Mitchell, 1996).

En la parte suroeste de la ciudad los intereses políticos del Estado, orientados por una fuerte demanda de viviendas básicas para grupos sociales de bajos ingresos, ha sido uno de los factores que explica los nuevos desarrollos inmobiliarios. Muchas de estas nuevas viviendas no alcanzan los $30 \mathrm{~m}^{2}$ de superficie para grupos familiares integrados, en promedio, por 5 personas, en sectores de la ciudad con carencias de urbanización, equipamiento, infraestructura de transporte y precarias condiciones ambientales (Olave, 1994). 
Figura 4. Distancia del cambio urbano entre los años 1943 y 2000.

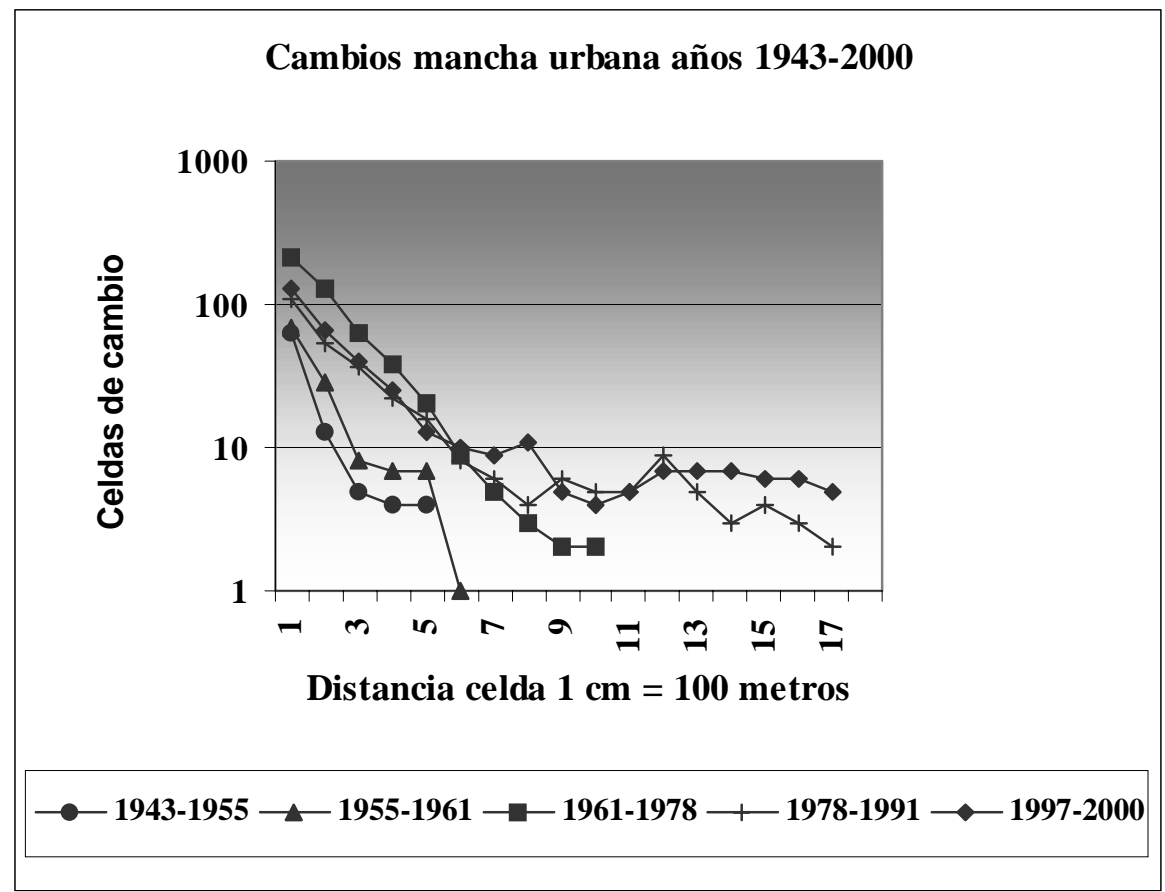

Figura 5. Patrón de cambio global entre 1943 y 2000.

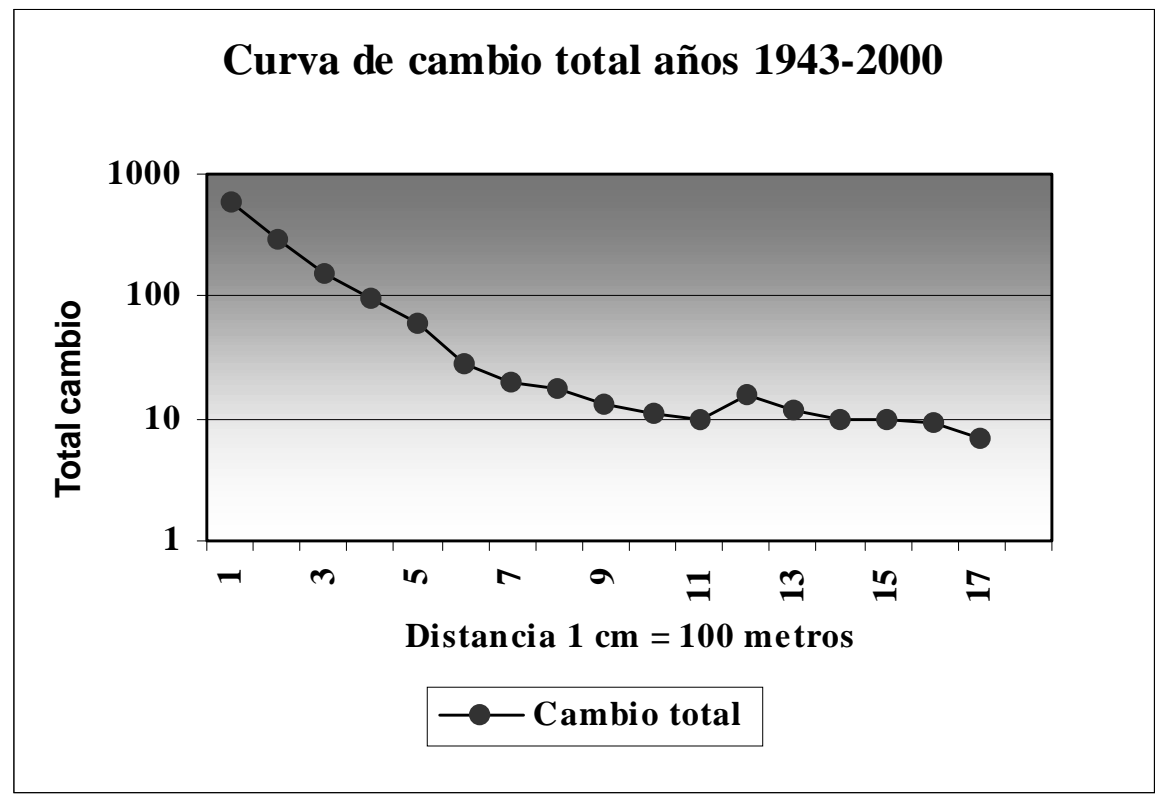




\subsection{Los patrones de crecimiento urbano y la condición socioeconómica de la población entre los años 1997 y 2000}

Entre los años 1997 y 2000, cerca de un $50 \%$ del cambio del uso del suelo en Chillán correspondió a nuevas zonas residenciales, y un $26 \%$ a uso industrial, siendo poco relevantes los demás usos del suelo en relación al cambio total. Las nuevas áreas residenciales de la ciudad fueron ocupadas por hogares pobres (37\%), hogares mixtos -es decir, familias clasificadas como pobres y no pobres$(32 \%)$, hogares no pobres (18\%) y hogares vulnerables (13\%). Del total de estas nuevas zonas residenciales, un $38 \%$ presentan densidades altas, entre 100-1.163 hab/há; un 26\% densidades medias, entre 50,1 y 100 hab/há, y un $36 \%$ densidades que van entre 0,1 y 50 hab/há.

En este período, la dinámica del cambio nos muestra la importancia del uso residencial en el crecimiento de la ciudad y la existencia de un proceso urbano de segregación espacial. Es así como el cambio urbano con altas densidades está asociado a zonas pobres y vulnerables, mientras el cambio con baja densidad, a zonas no pobres y segmentos de población de ingresos medios-altos. La población de ambas zonas presenta diferentes perfiles socioeconómicos, que a su vez reflejan diferentes patrones de localización en contextos específicos de paisaje.

También es importante destacar la existencia de un sector residencial, ubicado en el núcleo urbano de Chillán Viejo, que no ha sido impactado por los cambios recientes de uso del suelo y que ha mantenido una actividad agrícola tradicional. En esta zona urbana es posible observar notables diferencias con otros barrios de la ciudad, y también con los predios agrícolas colindantes. Estamos en presencia de un área de transición urbano-rural, caracterizada por la existencia de una actividad agrícola tradicional, un particular estilo arquitectónico y una forma de vida rural, asociada a la cultura y tradiciones del campo. En este sector la ciudad se prolonga hacia el mundo rural, o bien la ruralidad actúa dentro de lo urbano como un elemento de defensa y resistencia a cambios que puedan impactar su identidad y estructura espacial.

\subsection{La distribución espacial del cambio urbano}

El crecimiento urbano que hemos denominado concéntrico se observa en la parte sur y suroeste de Chillán, en torno a Chillán Viejo. En esta zona existen carencias de urbanización, como falta de pavimentación de calles, mal estado de luminarias y de señalética urbana y grandes sitios eriazos, resabios de un proceso de crecimiento urbano no planificado. Socialmente, son percibidas como áreas residenciales inseguras, y en su apropiación participan grupos sociales marginales (llustre Municipalidad de Chillán, 2000).

Cabe consignar que en esta parte de la ciudad no existen otros usos que hayan experimentado cambios importantes y que puedan servir de apoyo a las actividades residenciales, como el equipamiento y los servicios. Más bien, los grandes equipamientos se localizan en el núcleo urbano tradicional, en torno a las denominadas "Cuatro Avenidas", y en la parte norte, cercanos a nuevas áreas residenciales para sectores de ingresos medios-altos.

El crecimiento en torno a ejes o patrón tentacular se observa en el acceso norte a Chillán, en el acceso sur y en el camino a Coinueco. En los dos primeros sectores está asociado principalmente a uso industrial, almacenamiento y distribución de insumos agrícolas, y en el tercero, a uso residencial con equipamiento de apoyo para los nuevos barrios, especialmente comercio y educación.

Las formas de crecimiento urbano, asociadas a los procesos económicos, sociales y políticos más globales, han contribuido a modelar una ciudad con fuertes contrastes socia- 
les y con severos problemas ambientales. Más bien deberíamos hablar de tres ciudades en una: la primera denominada histórica o del casco antiguo, ubicada entre las cuatro avenidas y en Chillán Viejo; la "ciudad de los pobres", en la parte sureste; y la nueva ciudad segregada o "de las oportunidades", en la parte noreste. También debemos agregar a esta clasificación los enclaves industriales en los accesos norte y sur, asociados a ejes de transporte de importancia regional, y los islotes de ruralidad que aún subsisten dentro de los límites urbanos.

\section{Conclusiones}

El crecimiento de la ciudad de Chillán ha sido tensionado por factores regionales y locales, generando una morfología urbana que puede ser descrita en términos de anillos concéntricos y de ejes asociados a diferentes sectores de la ciudad, usos del suelo, zonas socioeconómicas, densidades de población y contextos específicos de paisajes. El desarrollo urbano de Chillán ha privilegiado, especialmente a partir de la década de los ' 80 , un continuo proceso de extensión de su superficie urbana construida, inclusive fuera de los límites urbanos, en desmedro de la densificación de zonas urbanas consolidadas y del centro histórico.

Los patrones del crecimiento urbano identificados en Chillán conservan algunas características de los modelos tradicionales que explican la evolución de las ciudades latinoamericanas, especialmente los anillos radiales y los ejes de crecimiento (Mertins, 2000). Sin embargo, aparecen nuevas estructuras y factores que dan cuenta de un proceso de crecimiento en que la dispersión y segregación del espacio urbano parecen haber aumentado, con una importancia cada vez mayor de factores locales en el crecimiento de la ciudad. La movilidad de la población y las preferencias de las personas, guiadas por su status y situación socioeconómica, junto a la oferta pública y privada de nuevos espacios residenciales, constituyen importantes factores en las decisiones de localización (Bryant et al., 1982). En otras palabras, las decisiones de localización en las nuevas áreas residenciales están siendo determinadas por una oferta inmobiliaria fuertemente segregada y por las preferencias individuales de las personas, reflejando los cambios en el uso del suelo la existencia de mercados segmentados que tienden a maximizar las distancias físicas como expresión del creciente distanciamiento social.

Por otra parte, la influencia de las políticas habitacionales del Estado, a través de la construcción de viviendas sociales y de la erradicación de asentamientos irregulares ubicados dentro de zonas urbanas consolidadas, ha fomentado el crecimiento en zonas periféricas, contribuyendo a la formación de espacios urbanos claramente segregados. Tal como señalan Marcuse y Van Kempen (2000), el mercado inmobiliario no ofrece muchas oportunidades de elección a las clases de menores ingresos, y de este modo, se va creando en Chillán una división social del espacio urbano. En algunos casos, estas agregaciones de nuevos usos residenciales -y también industriales- superan los actuales límites urbanos, dando origen a un mosaico de usos del suelo con paños industriales, residenciales y agrícolas poco integrados a la vialidad estructurante. Se produce una segmentación socioespacial de la ciudad con nuevas áreas urbanas, especialmente condominios y parcelaciones residenciales de $5.000 \mathrm{~m}^{2}$

En ambos casos, detrás del movimiento de población y el surgimiento de nuevos desarrollos inmobiliarios, está la acción de privados y del Estado, pero no siempre con una visión estratégica del significado urbano-ambiental de estos nuevos emprendimientos y de sus factores causales. Lo anterior, indudablemente, nos permite concluir que el desarrollo urbano de Chillán ha sido espontáneo e inorgánico y la ciudad muestra marcadas diferencias morfológicas, con problemas de integración y comunicación entre los distintos sectores y barrios. No existe una relación funcional entre 
las sub-ciudades o barrios identificados; cada área mantiene sus especificidades y reproduce su propio desarrollo, como también se vincula de manera diferente con el entorno rural inmediato. No existen elementos urbanos que den continuidad a la ciudad, sino más bien hay ciertos elementos estructurantes que actúan como barrera al crecimiento e integración urbana, como son la vía férrea y el Estero Las Toscas.

En síntesis, y de acuerdo a los planteamientos teóricos de esta investigación (McDonal \& McMillen, 1998; Newman et al., 1992; Young, 1995), podemos afirmar que la morfología y funcionalidad urbana de Chillán son, actualmente, el resultado de una serie de procesos espontáneos e inorgánicos gatillados por factores regionales y locales que han contribuido al desarrollo de una ciudad morfológica y socialmente fragmentada. Respecto a las variables que explican estos nuevos patrones de crecimiento urbano, podemos mencionar la accesibilidad a la vialidad estructurante, la posición geográfica de Chillán respecto al sistema de asentamientos urbanos de la provincia de Ñuble, las características socioeconómicas de la comunidad local y la política inmobiliaria del Estado y de los privados, como los principales factores que explican la evolución de la superficie urbana construida, los cambios en el uso del suelo y los diferentes patrones identificados.

Finalmente, no se observa en el desarrollo urbano de la ciudad una sincronía en el accionar de los actores públicos y privados que a futuro permita proyectar cambios importantes en las actuales tendencias de crecimiento detectadas.

\section{Referencias bibliográficas}

Azócar, G. \& M. Muñoz (1998). "Estructura territorial y uso del suelo urbano". Parra, $\mathrm{O}$. \& E. Habit (eds.), Estudio de línea de base para la evaluación de impacto ambiental del complejo forestal industrial Itata (Celu-
Iosa Arauco y Constitución S.A.). Concepción: Editora Aníbal Pinto S.A.

Azócar, G., H. Romero, R. Sanhueza, M. D. Muñoz y C. Vega (2003). "Los patrones de urbanización en ciudades intermedias chilenas y sus impactos sobre el reordenamiento social del espacio urbano; un caso de estudio en Santa María de Los Ángeles, Chile Central" (manuscrito en elaboración).

Barber, B. (2000). "Vers une societe universelle de conssommateurs. Culture McWorld contre democratie". Elbaz, M. \& D. Helly (eds.), Mondialization, citoyennete et multiculturalisme. Quebec: Les Presses de'l Universite de Laval.

Bryant, C., L. Russwurm y A. McLellan (1982). The Cities Countryside: Land and its management in the rural urban fringe. Londres: Longman.

Boisier, S. (2001). "Crónica de una muerte frustrada: el territorio en la globalización”. Notas del expositor para una exposición. www.ider.cl/archivos/Boisier,\%20dos.pdf

Borsdorf, A. (2000). "El desarrollo urbano de Valdivia. Estudio de Caso en una ciudad mediana chilena". Espacio y Desarrollo, 12: 45-82.

De Mattos, C. (2001). "Metropolización y suburbanización”. EURE, 27, 80: 5-8.

González, S., D. Olave y E. Vega (1994). "Algunos problemas ambientales que inciden en la calidad de vida en la ciudad de Chillán”. Tiempo y Espacio, 4: 113-118.

Hanhörster, H. (2001). "Whose neighbourhood is it? Ethnic diversity in urban spaces in Germany". Geojournal, 51:329-338.

Henríquez, C. \& G. Azócar (2001). "El Componente Socio-Económico en la Sustentabilidad Ambiental de Ciudades Intermedias en Chile Central; Estudio de la ciudad de Chillán". III Congreso Internacional de Ordenación del Territorio, Gijón (España).

Hervieu, B. \& J. Viard (1996). Au bonheur des campagnes (et des provinces). París: Ediciones de l' Aube, La Tour d' Aigües.

llustre Municipalidad de Chillán (2000). Plan de Desarrollo Comunal PLADECO, 19982000, Chillán. 
INE (1992). Resultados Generales. Censo de Población y Vivienda. Chile. Santiago: INE.

INE \& MINVU (2001). Cifras preliminares PreCenso de Población y Vivienda año 2001, Región del Biobío (documento de trabajo).

López, E., G. Bocco, M. Mendoza y E. Duhau (2001). "Predicting land-cover and land-use change in the urban fringe: A case in Morelia city, Mexico". Landscape and Urban Planning, 55: 271-285.

Marcuse, P. \& R. Van Kempen (eds.) (2000). Globalizing cities: A new spatial order. Oxford: Blackwell.

McDonal, J. \& D. McMillen (1998). "Employment subcenters and subsequent real state development in suburban Chicago". Brooking-UIC Sprawl Proyect.

Mertins, G. (2000). "Ciudades medianas en América Latina: criterios, indicadores y el intento de un modelo de su diferenciación socio-espacial y funcional". Espacio y Desarrollo, 12: 123-140.

Newman, P., J. Kenworthy y P. Vintila (1992). Housing, Transport and urban form. Perth: Institute for Science and Technology Policy, Murdoch University.

Olave, D. (1994). "La vivienda una variable de calidad de vida en la ciudad de ChillánChile". Tiempo y Espacio, 4: 95-112.

(1996). "Calidad de vida en la viudad de Chillán-Chile". Tiempo y Espacio, 6: 61-73.

Olave, D., M. Mardones y S. González (1996). "Niveles de calidad de vida en la Ciudad de Chillán". Tiempo y Espacio, 6: 66-74.

Parker, A. (1995). "Patterns of federal urban spending: central cities and their suburbs, 1983-1992". Urban affairs Review, 31, 2: 184-205.

Romero, H. \& X. Toledo (1998a). "Crecimiento económico y desigualdades regionales en Chile; indicadores de evaluación ambiental estratégica". Anales de la Sociedad Chilena de Ciencias Geográficas: 537-570. (1998b). "Crecimiento econó- mico y comportamiento espacial del sector inmobiliario en Chile". Revista Geográfica de Chile Terra Australis, 43: 131-203.

(2000). "Ecología urbana y sustentabilidad ambiental de las ciudades intermedias chilenas". Anales de la Sociedad Chilena de Ciencias Geográficas: 445452.

Rovira, A. (2000). "Puerto Montt: El paso de una ciudad menor a centro de desarrollo interregional en una ciudad del sur de Chile". Espacio y Desarrollo, 12: 83-102.

Sabatini, F. (1998). "Direcciones para el futuro". Jordán, R. \& D. Simioni (comps.), Ciudades intermedias en América Latina y el Caribe: Propuestas para la gestión urbana. Santiago: CEPAL/Ministero degli Affaire Esteri Cooperazione Italiana, 127-214.

Thomson, L. M. \& C. J. A. Mitchell (1996). "Counterurbanization in Waterloo Region: the lure of the countryside". Filion, P., T. Bunting y K. Curtis (eds.), The Dynamic of the Dispersed City: Geographic and Planning Perspectives on Waterloo Region. Waterloo: Department of Geography Publications Series, 47: 303-328.

Vink, A. P. A. (1982). "Anthropocentric landscape ecology in rural areas". Tjallingii, S. P. \& A. A. de Veer (eds.), Perspectives in Landscape ecology. Contributions to research, planning and management of our environment. Wageningen: Center for Agricultural Publishing and Documentation, 87-98.

Wiegand, T. (2001). "Programita, A sofware to perform point pattern analysis with Ripleys $\mathrm{L}$ and the O-Ring statistic". http:// pinus.oesa.ufz.de/towi/

Young, D. (1995). Alternatives to Sprawl. Cambridge, MA.: Lincoln Institute of Land Policy.

Zhang, T. (2001). "Community features and urban sprawl: the case of the Chicago metropolitan region". Land Use Policy, 18: 221-232. 